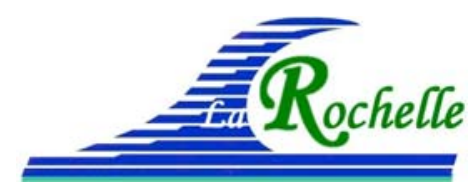

XVèmes Journées Nationales Génie Côtier - Génie Civil

La Rochelle, 29 au 31 mai 2018

DOI:10.5150/jngcgc.2018.059 @ Editions Paralia CFL

disponible en ligne - http://www.paralia.fr - available online

\title{
Le Geocorail : un matériau innovant pour la lutte contre l'érosion et le renforcement d'ouvrage maritime
}

\author{
Alaric ZANIBELLATO ${ }^{1}$, Sébastien BIGARE ${ }^{1}$, Nicolas VERJAT ${ }^{1}$, \\ Philippe ANDREANI ${ }^{1}$, Jérôme SOLESIO ${ }^{1}$ \\ 1. Geocorail SAS, 4 rue Gaston Castel, 13016 Marseille, France. \\ alaric.zanibellato@geocorail.com
}

\section{Résumé :}

Face aux phénomènes d'érosion marine de plus en plus sévères ces dernières années, des solutions pérennes doivent être mises en place pour renforcer les infrastructures portuaires, limiter le recul des dunes, plages, falaises et assurer la sécurité des résidents. Dans ce contexte, la société Geocorail SAS développe et commercialise un procédé électrochimique permettant de produire un agglomérat rocheux en milieu marin, à partir des matériaux présents sur place tels que sable, gravier ou débris coquilliers. Ce "béton naturel", solide et extrêmement adhésif permet de réparer ou de consolider des structures immergées ou semi-immergées.

Mots-clés :

Littoral, Erosion, Procédé électrochimique, Geocorail, Protection.

\section{Introduction}

On ne peut évoquer le contexte scientifique de l'activité de Geocorail sans établir de lien avec la géographie et le problème d'érosion côtière.

A ce propos, rappelons simplement quelques chiffres qui résument bien la situation (COLAS, 2007) :

- 24\% de littoral en France recule du fait de l'érosion marine, soit $1723 \mathrm{~km}$ sur $7124 \mathrm{~km}$.

- 2/3 de ces côtes en recul sont sableuses, 1/3 est de nature rocheuse ou solide.

- Les communes littorales maritimes accueillent 10\% de la population métropolitaine, soit environ 7 millions d'habitants.

- A ces 7 millions de résidents, il faut ajouter les 7 millions de lits touristiques (hôtellerie, camping, résidences secondaires), situés sur le littoral.

- $25 \%$ du littoral situé à moins de $500 \mathrm{~m}$ de la mer est urbanisé.

Le littoral français est parsemé d'infrastructures et d'ouvrages maritimes indispensables

à l'activité économique, dont :

- 149 ports ;

- 109 ports-abris $(<0.5$ ha et sans capitainerie) ;

- 137 terre-pleins ;

- 62 plages alvéolaires ; 


\section{Thème 4 - Ouvrages portuaires, offshore et de plaisance}

- 397 épis ;

- 35 endigages d'embouchures de cours d'eau.

Ces ouvrages représentent autant d'investissements lourds des collectivités et de l'Etat, qui nécessitent une maintenance continue et des restaurations coûteuses.

La société concentre son activité autour du développement et de la commercialisation de procédés et techniques innovants permettant d'allonger la durée de vie d'ouvrages maritimes neufs ou de consolider et réparer des ouvrages maritimes défaillants.

Elle poursuit également une $R \& D$ fondamentale et appliquée autour des problématiques de capture des polluants (CARRE et al., 2017) et de restauration des biotopes.

\section{Processus de formation du Geocorail}

Le procédé de formation du Geocorail est le suivant: en appliquant un courant cathodique très basse tension à travers une structure métallique immergée sous des sédiments marins, la réduction de l'oxygène dissous et/ou la réduction de l'eau conduisent à une augmentation du $\mathrm{pH}$ interfacial et à la précipitation de carbonate de calcium et d'hydroxyde de magnésium (BARCHICHE et al., 2003). Ces deux composés, l'aragonite $\left(\mathrm{CaCO}_{3}\right)$ et la brucite $\left(\mathrm{Mg}(\mathrm{OH})_{2}\right)$, créent un liant, sorte de "ciment naturel" et favorisent la sédimentation des grains de sables, gravillons, débris coquillers et autres élément transportés par les vagues pour former un matériau solide, rugueux et très cohésif autour de la cathode (DANG NGUYEN et al., 2017), (figure1).

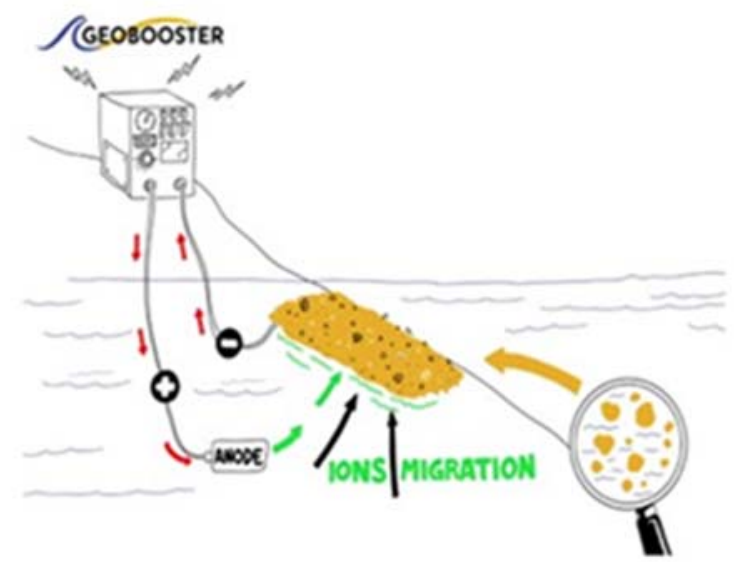

Figure 1. Schéma du procédé de fabrication du Geocorail.

A ce jour, les cinétiques ont permis d'obtenir des épaisseurs de l'ordre $5 \mathrm{~cm}$ en 6 mois (fig.2). L'épaisseur maximale obtenue a été de $50 \mathrm{~cm}$ en 18 mois.

La mise en œuvre du dispositif ne nécessite pas d'apports de matériaux, de travaux d'infrastructures et de terrassements majeurs, sauf à réaliser la pose et l'ancrage du support, et l'effacement des câbles d'alimentation et de mesure. 


\section{XVèmes Journées Nationales Génie Côtier - Génie Civil \\ La Rochelle, 29 au 31 mai 2018}
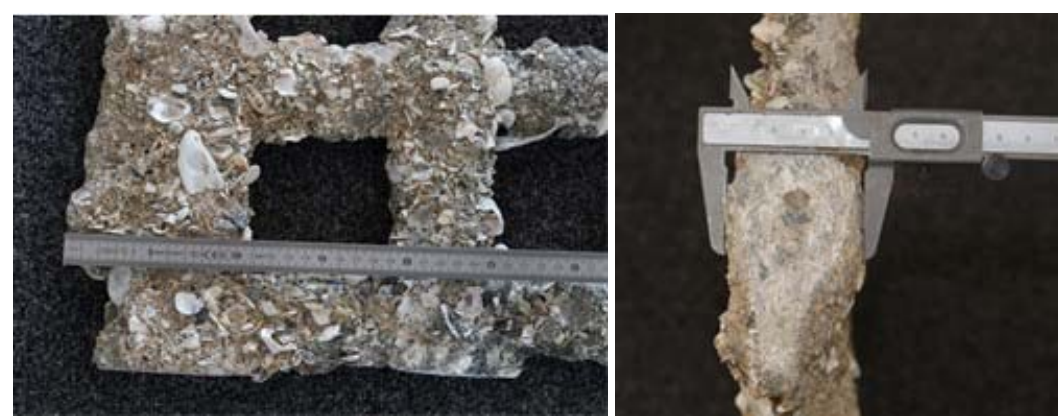

Figure 2. Exemple d'un morceau de Geocorail de 14 mois formé sur un treillis soudé $15 \times 15 \times 0.7 \mathrm{~cm}$ sur le Chantier Pilote à Châtelaillon-Plage (17).

Il est important de rappeler que la cinétique de croissance et les caractéristiques mécaniques du Geocorail sont entièrement dépendantes des facteurs environnementaux (granulométrie du site, température de l'eau, conditions hydrodynamiques...) et des valeurs de courant injectés (cœur du process de Geocorail SAS).

Afin d'optimiser la croissance et la solidité du matériau, un laboratoire de R\&D comprenant 40 bacs autonomes a été mis en place depuis septembre 2016 à Fos sur Mer pour comprendre et appréhender le plus finement possible l'influence des différents paramètres (figure 3).

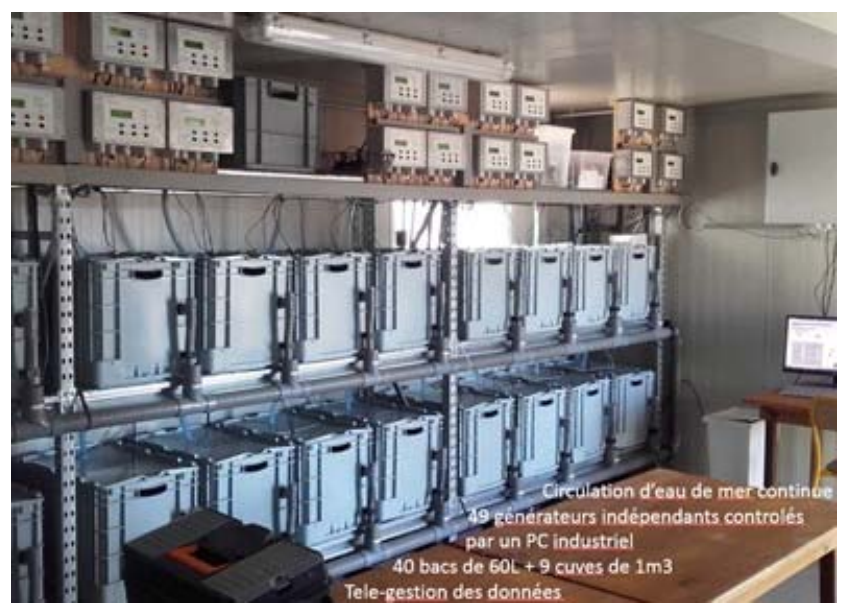

Figure 3. Laboratoire R\&D de Fos-sur-Mer.

L'entreprise Geocorail est intégrée dans le programme ANR Ecocorail depuis 2013, en partenariat avec les universités de La Rochelle, de Caen-Basse Normandie et de Nouméa. Dans le cadre de ce programme, une première approche de caractérisation mécanique a été menée avec le département Génie Civil de La Rochelle. Des essais ont été réalisés sur des blocs de Geocorail formés à Belle-île en Mer dans les années 1990. Ces tests montrent une résistance à la compression de l'ordre de $10 \mathrm{MPa}$, soit l'équivalent d'un mortier. Un stage va commencer en mai 2018 à l'IUT Génie Civil de La Rochelle pour continuer cette démarche, mais cette fois-ci sur des matériaux formés 


\section{Thème 4 - Ouvrages portuaires, offshore et de plaisance}

à Chatelaillon-Plage, prélevés plusieurs fois par an pendant 3 ans. Ces essais permettront d'étudier l'évolution du matériau dans le temps et de prédire son comportement futur. L'entreprise fait également partie du projet B-Corail qui réunit l'université de La Rochelle (Département Génie-Civil et Département de Chimie), Planète Recyclage SAS et l'Entreprise Charrier. Ce projet a pour objectif d'étudier la formation du matériau dans des granulats de béton recyclé, en laboratoire et In-Situ.

\section{Applications}

Les supports d'enrochements

- Principe de base : En appui des méthodes traditionnelles, lors de travaux création ou d'entretien, pose d'un support sous les enrochements à venir pour renforcer l'ensemble de la structure

- Bénéfices de la solution: Impact financier minime sur l'opération, Temps d'intervention extrêmement réduit, accroissement de la durée de vie, diminution des coûts d'entretien, disparition des phénomènes d'affouillement (fig. 4 et 5);

- Amélioration à venir : étude en cours, en canal à houle, pour envisager la diminution de la quantité de blocs nécessaires tout en garantissant au moins la même performance de l'ouvrage.
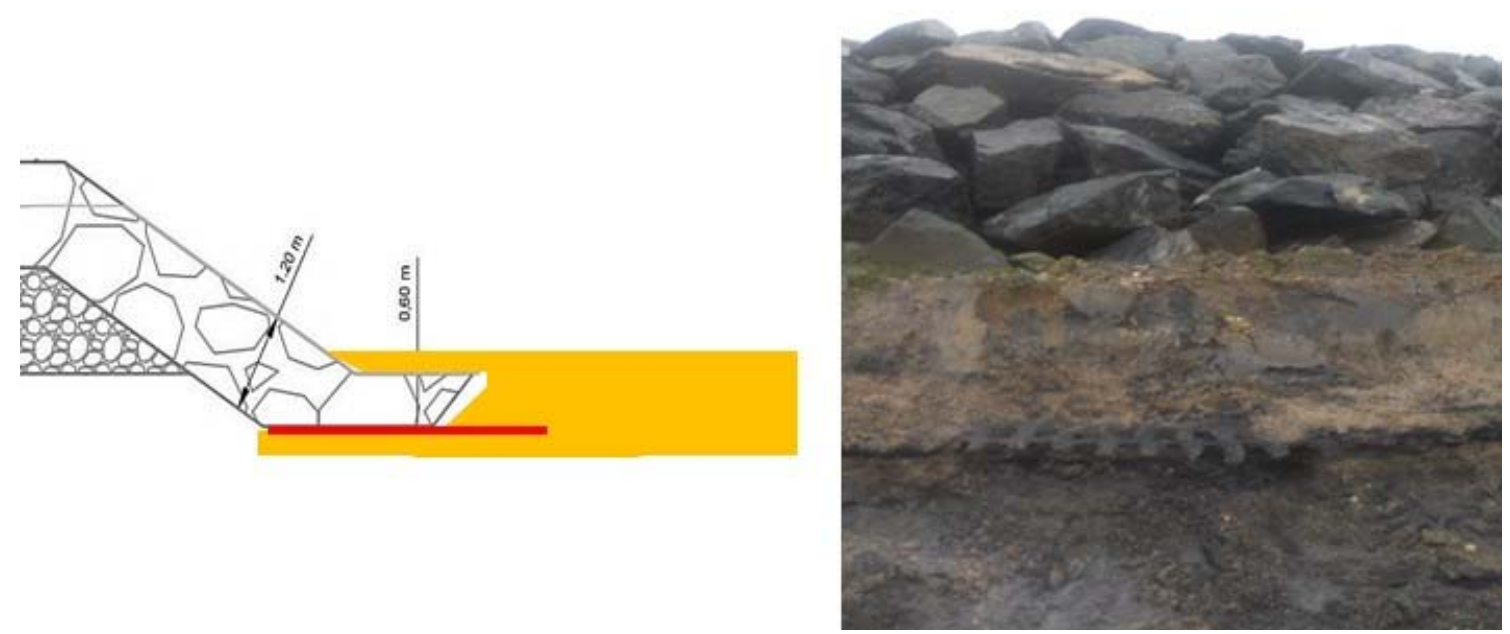

Figure 4. Application pour un support d'enrochement.

\section{L'anti affouillement}

- Principe de base : En pied de falaise ou sous un quai, par exemple, nous comblons l'affouillement existant et posons en applique un support que nous renforçons en Geocorail®,

- Bénéfices de la solution : invisible à l'œil (vs béton projeté), possibilité de déployer la solution au-delà du point affouillé pour éviter un futur nouveau problème, impact écologique neutre, accroissement de la durée de vie de l'ouvrage, 


\section{XVèmes Journées Nationales Génie Côtier - Génie Civil \\ La Rochelle, 29 au 31 mai 2018}

- Amélioration à venir : possibilité de déployer une solution en géotextile mixte dont l'effet de retenue est immédiat.

Le confortement de berges et les géotubes

- Principe de base : En se servant des propriétés du Geocorail ${ }^{\circledR}$, pose de géotextile mixte pour maintenir les berges, diminuer les quantités de matières draguées, mettre en place des géotubes plus résistants.

- Bénéfices de la solution: Réduction des volumes de dragage, disparition risque percement des géotubes, accroissement durée de vie des ouvrages, diminution coûts d'entretien, facilité de mise en œuvre du produit, mise en forme sur mesure (fig.5),

- Amélioration à venir: Développement en cours d'une solution électriquement autonome.

La séquestration des polluants

- Principe de base: En déployant sur le fond le géotextile mixte, nous encapsulons immédiatement les polluants. En créant le Geocorail ${ }^{\circledR}$, nous séquestrons les sédiments pollués sous une coque solide sans risque de dispersion dans la colonne d'eau.

- Bénéfices de la solution : Peu de moyens logistiques : mise en œuvre simple, durée d'intervention très courte, efficacité immédiate par la pose du géotextile, pas de diminution du tirant d'eau, coûts de déploiement très faibles

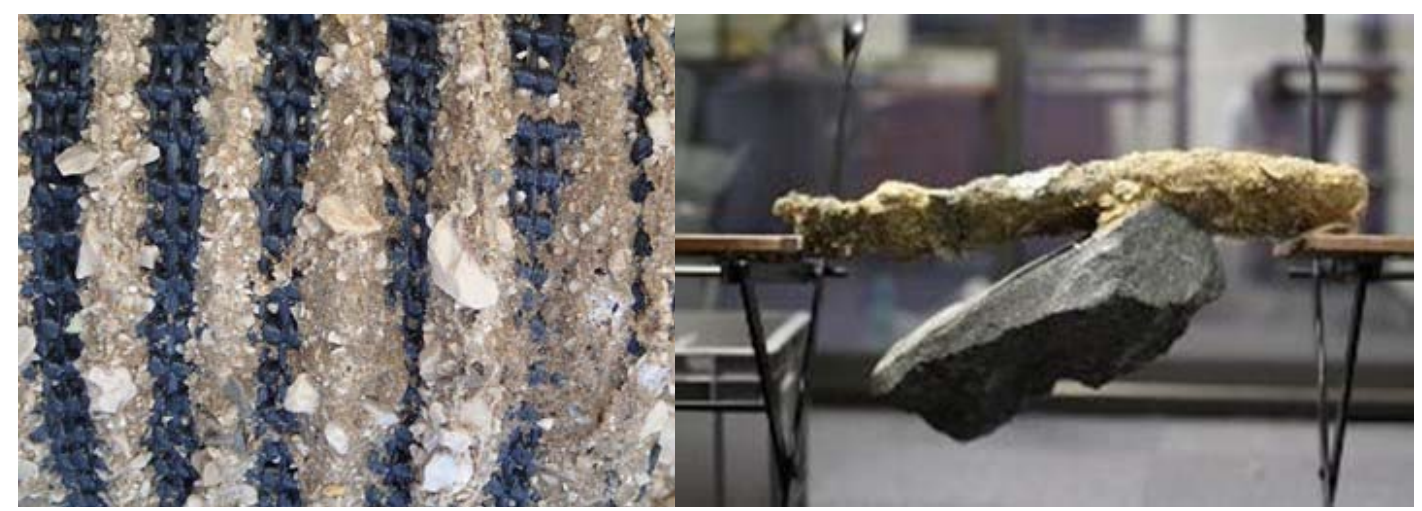

Figure 5. Exemples d'application du Geocorail : renforcement de geotextile (image de gauche) et prise en masse rocher (Durite, $8 \mathrm{~kg}$ )- Geocorail (image de droite).

La restauration écologique

- Principe de base : Réalisation sur mesure de structures en 3D ou à façon visant à favoriser le retour de la faune et la flore endémiques.

- Bénéfices de la solution: Créations sur mesure $=>$ pas de limite, possibilité de préfabrication avant mise en place, applications multiples : spots de plongée, Ecoconception, corps morts, ..., intervention en parallèle des chantiers structurels, propriétés identiques au milieu marin $=>$ repeuplement, $\mathrm{pH}$ identique à celui de la mer \& rugosité du matériau (fig.6) 


\section{Thème 4 - Ouvrages portuaires, offshore et de plaisance}

- Amélioration à venir: Etude environnementale en cours pour quantifier la performance de notre solution.

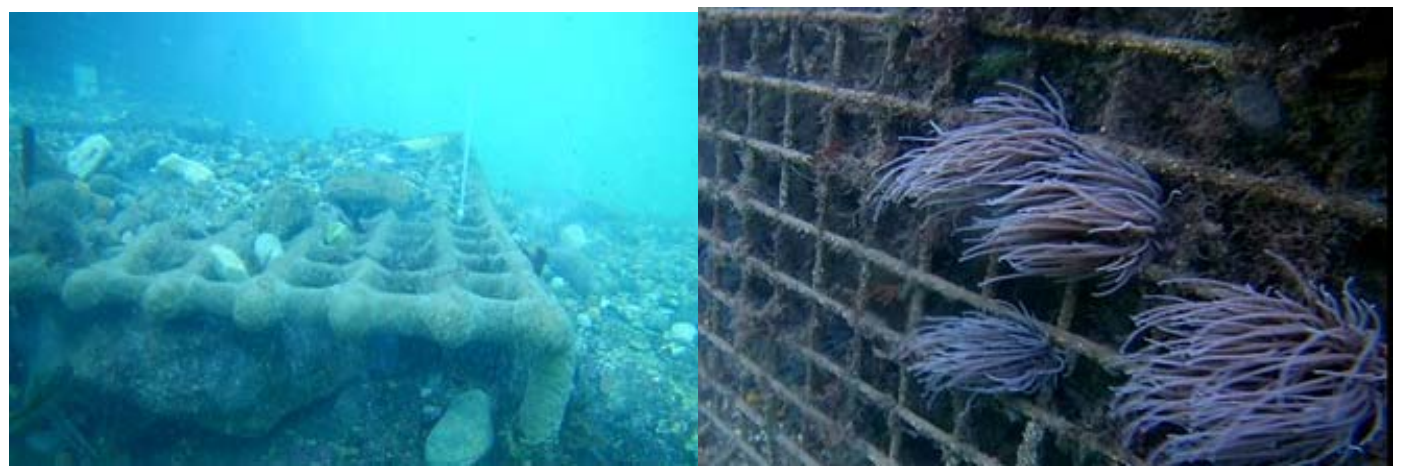

Figure 6. Tapis anti-affouillement Geocorail installé en pied de quai, Le Pradet, 2017.

\section{Limites et atouts de la technologie Geocorail ${ }^{\circledR}$}

Limites :

- Le temps nécessaire à la concrétion du matériau est incompatible avec les travaux d'urgence

- La présence de sédiments est nécessaire à la formation de ce béton naturel ; dans la colonne d'eau, seul un dépôt calco-magnésien sera formé.

- S’il n’y a que des vases très fines, le procédé fonctionne mal (absence de renouvellement d'eau), il faut donc ajouter d'autres sédiments

- A l'heure actuelle des études sont en cours pour caractériser les propriétés de résistance, flexion, adhésivité du matériau. Pour l'instant, nous n'avons que peu d'indicateurs fiables.

- En termes de restauration écologique, nous voyons que le matériau est attractif pour la faune et la flore mais les études à venir affineront les données.

Atouts :

- La technologie se décline en de multiples applications

- Geocorail a la capacité de réaliser des ouvrages sur mesure

- Technique écologiquement douce mais structurellement efficace, avec une consommation énergétique de quelques watt $/ \mathrm{m}^{2}$

- Quelle que soit l'application, le coût de déploiement de la solution est faible par rapport au projet global

- Dans le cadre d'applications structurelles, le maitre de l'ouvrage réalisera d'importantes économies d'entretien de son patrimoine. Le coût moyen d'entretien d'un enrochement sur la durée de vie de l'ouvrage est compris entre $3 \%$ et $5 \%$ de son coût de construction neuf par an (Ministère de l'environnement, de l'énergie et de la mer, 2017). 


\section{XVèmes Journées Nationales Génie Côtier - Génie Civil \\ La Rochelle, 29 au 31 mai 2018}

\section{Perspectives}

La société Geocorail est aujourd'hui en phase de commercialisation. Des projets de support d'enrochement sont en cours, notamment en Martinique, à La Réunion, et en Polynésie Française, portant sur un montant global de plus d'un million d'euros.

Au-delà des applications existantes de renforcement de structures marines, deux axes de développement sont approfondis :

- le GEOCAPTAGE, qui a pour but le captage de polluants dans les ports. Le projet Geocaport, financé par l'ADEME et le GPMLR est en cours depuis 2016, au niveau de la base sous-marine du Port Atlantique.

- GEORESTORE, qui a pour objectif la restauration écologique des petits fonds côtiers.

Geocorail SAS avance avec plusieurs partenaires dans cette démarche de R\&D, tant les sujets sont vastes et à la croisée de plusieurs champs de compétence (mécanique, géniecivil, hydrodynamique, chimique, électrique, électrochimique, biologique, toxicologique...)

Une attention particulière est portée depuis 2016 sur la caractérisation mécanique du matériau formé. Deux projets universitaires sont axés sur cette thématique :

- 2017-2018 (13 mois) : Post-doctorat à l'Ecole des Arts et Métier d'Aix en Provence, dirigé par Agnès Fabre. Etude des propriétés mécaniques du Geocorail ${ }^{\circledR}$ formé en laboratoire à Fos-sur-Mer

- Mai-Juin 2018 : Stagiaire à l'IUT Génie-Civil de l'Université de la Rochelle, encadré par Pierre-Yves Mahieux. Etude portant sur les propriétés mécaniques du Geocorail formé en site naturel (Chatelaillon-plage)

Ces projets permettront de comparer le Geocorail formé à des matériaux connus dans le Génie Civil (mortier, béton, roche...).

Enfin, une réunion organisée par la mairie de Châtelaillon-Plage aura lieu en Septembre 2018. Cette réunion va réunir différents acteurs liés au littoral (DDTM, DREAL, CEREMA, CREOCEAN, EGIS PORT, UNIMA, ARTELIA, Région Nouvelle Aquitaine, Département Charente Maritime, GPMM...) afin de discuter des moyens d'applications de ce procédé dans les prochaines années en Charente-Maritime.

\section{Références}

BARCHICHE C., DESLOUIS C., FESTY D., GIL O., REFAIT P., TOUZAIN S., TRIBOLLET B. (2003). Characterization of calcareous deposits in artificial seawater by impedance techniques: 3-Deposit of CaCO3 in the presence of $\mathrm{Mg}(\mathrm{II})$. Electrochimica Acta, Vol. 48(12), pp 1645-1654. https://doi.org/10.1016/S0013-4686(03)00075-6 CARRE C., GUNKEL-GRILLON P., SERRES A., JEANNIN M., SABOT R., QUINIOU T. (2017). Calcareous electrochemical precipitation, a new method to trap nickel in seawater. Environmental Chemistry Letters, Vol. 15(1), pp 151-156. https://doi.org/10.1007/s10311-016-0602-2 
Thème 4 - Ouvrages portuaires, offshore et de plaisance

CETMEF (2009). Guide Enrochement - L'utilisation des enrochements dans les ouvrages hydrauliques.

COLAS S. (2007). Analyse statistique et cartographique de l'érosion marine, Institut Français de l'Environnement, Les dossiers de l'IFEN, - ${ }^{\circ} 6$.

DANG NGUYEN D., GASCOIN S., ZANIBELLATO A., DA SILVA C. G., LEMOINE M., RIFFAULT B., SABOT R., JEANNIN M., CHATEIGNER D., GIL

O. (2017). Role of brucite dissolution in calcium carbonate precipitation from artificial and natural seawaters. Crystal Growth \& Design, Vol. 17(4), pp 1502-1513. https://doi.org/10.1021/acs.cgd.6b01305 